\title{
Tetraphenylethylene-BODIPY aggregation-induced emission luminogens for near-infrared polymer light-emitting diodes
}

\author{
Sebnem Baysec ${ }^{1}$, Alessandro Minotto ${ }^{2}$, Patrick Klein ${ }^{1}$, Simone Poddi ${ }^{2}$, Andrea Zampetti ${ }^{2}$, \\ Sybille Allard ${ }^{1}$, Franco Cacialli ${ }^{2}$ \& Ullrich Scherf ${ }^{1 *}$ \\ ${ }^{1}$ Macromolecular Chemistry and Institute for Polymer Technology, Bergische Universität Wuppertal, Wuppertal, 42097 Germany \\ ${ }^{2}$ Department of Physics and Astronomy and London Centre for Nanotechnology, University College London, London, WC1E 6BT, UK
}

Received April X, 2018; accepted XX, 2018; published online XXX

\begin{abstract}
The Aggregation-Induced Emission (AIE) phenomenon provides a new direction for the development of organic lightemitting devices. Here, we present a new class of emitters based on 4,4-difluoro-4-bora-3a,4a-diaza-s-indacene (BODIPY), functionalized at different positions with tetraphenylethylene (TPE), which is one of the most famous AIE luminogens. Thanks to this modification, we were able to tune the photoluminescence of the BODIPY moiety from the green to the near-infrared (NIR) spectral range and achieve PL efficiencies of $\sim 50 \%$ in the solid state. Remarkably, we observed an enhancement of the AIE and up to $\sim 100 \%$ photoluminescence efficiencies by blending the TPE-substituted BODIPY fluorophores with a poly[(9,9di- $n$-octylfluorene-2,7-diyl)-alt-(benzo[2,1,3]thiadiazol-4,7-diyl)] (F8BT) matrix. By incorporating these blends in organic lightemitting diodes (OLEDs), we obtained electroluminescence peaked in the range $650-700 \mathrm{~nm}$ with up to $1.8 \%$ external quantum efficiency and $\sim 2 \mathrm{~mW} / \mathrm{cm}^{2}$ radiance, a remarkable result for red/NIR emitting and solution-processed OLEDs.
\end{abstract}

BODIPY, Organic Light-Emitting Diode, Aggregation-Induced Emission, NIR Emission, Photoluminescence, Electro luminescence

Citation: Baysec S, Minotto A, Klein P, Poddi S, Zampetti A, Allard S, Cacialli F, Scherf U. Tetraphenylethylene-BODIPY aggregation-induced emission luminogens for near-infrared polymer light-emitting diodes.

\section{Introduction}

Recent developments in many fields of modern science and medicine show that oligomeric and polymeric dyes absorbing or emitting in the red/near-infrared (NIR) region $(650-900 \mathrm{~nm})$ have great importance [1-3]. The NIR dye can be either attached as a side chain or incorporated into the oligomer or polymer backbone. The main advantages of such dyes are a high homogeneity of the resulting active layer, an enhanced processability and high stability [4].

Among several organic fluorescent dyes, BODIPY (4,4'-difluoro-4-bora-3a,4a-diaza-s-indacene) still retains popularity due to its excellent spectroscopic features such as a high absorption coefficient, narrow absorption and emission bands, high fluorescence quantum yields in solution $\left(\Phi_{\mathrm{F}}>0.5\right)$, and excellent chemical and photostability [5-7]. Furthermore, the emission band can be spectrally tuned from the green to the NIR spectral range by chemical modification of the BODIPY molecular core. However, as most other organic dyes, BODIPY and its derivatives suffer from aggregation quenching and small Stokes shifts which limits their applications in organic electronics, for instance in organic light-emitting diodes (OLEDs). 
A promising approach to improve the photophysical properties of BODIPY is to promote Aggregation-Induced Emission (AIE) behavior by incorporating the most prominent member of the AIE organic luminogen family: TPE (tetraphenylethylene). The AIE-active luminogen shall improve the solid-state emission properties of BODIPY by overcoming the AggregationCaused Quenching (ACQ) problem while maintaining the already mentioned advantages of the BODIPY chromophore [8-13]. Design and application of AIE luminogens are a hot research topic since the AIE phenomenon was first reported in 2001 by Tang and co-workers [14]. AIE luminogens are poorly emissive in dilute solutions but form highly emissive aggregates in the condensed state, e.g. either by addition of a non-solvent or in the solid state. The AIE effect is mainly due to the propellershape structure of the luminogens, which cause restriction of intramolecular rotations upon aggregation [15].

To the best our knowledge, only few articles [16-23] have investigated the substitution of BODIPY with varying numbers of AIE active aryl substituents in different positions, with substitution in 8-position of BODIPY allowing for the most distinct variation of the aggregated state emission.

In this context, our study provides a systematic investigation of substitution effects (alkyl substituents, number of TPE groups) and their consequences on the OLED performance. The materials exhibit $\Phi_{\mathrm{F}}$ reaching up to $50 \%$ in the solid state as neat thin films and up to $100 \%$ when embedded in a blend with poly[(9,9-di- $n$-octylfluorene-2,7-diyl)-alt(benzo[2,1,3]thiadiazol-4,7-diyl)] (F8BT). By sandwiching such blends between conventional electrodes, we obtained OLEDs exhibiting electroluminescence (EL) peaked in the range 650- $700 \mathrm{~nm}$, with up to $1.8 \%$ external quantum efficiency (EQE) and $2 \mathrm{~mW} / \mathrm{cm}^{2}$ radiance, which are among the highest values reported so far for fully solution-processed red/NIR emitting OLEDs not leveraging phosphorescence.

\section{Experimental}

\subsection{Synthesis}

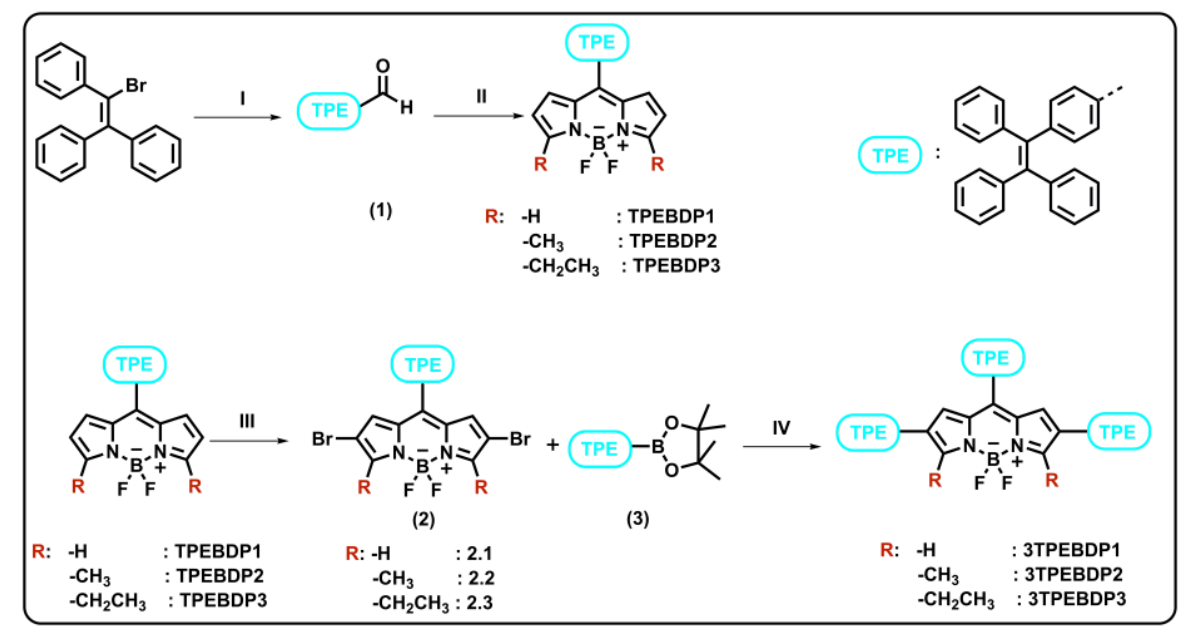

Scheme 1. Synthesis route to TPE substituted BODIPYs (I: (4-formylphenyl) boronic acid, TBAB, $\mathrm{Pd}\left(\mathrm{Ph}_{3}\right)_{4}$, toluene. II:

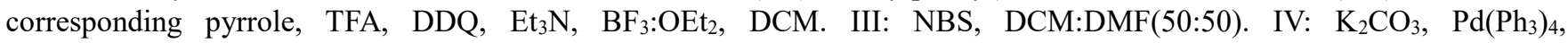
THF:water(80:20)).

The starting materials (2-bromo-1,2-diphenylvinyl) benzene and 4-formylphenylboronic acid were obtained from commercial suppliers and used without further purification. The obtained triphenylvinylbenzaldehyde (1) was condensed with the corresponding pyrrole in the presence of trifluoroacetic acid (TFA), followed by oxidative dehydrogenation with 2,3dichloro-5,6-dicyano-1,4-benzoquinone (DDQ) and complexation with boron trifluoride diethyl etherate $\left(\mathrm{BF}_{3}\right.$ : $\left.\mathrm{OEt}_{2}\right)$ to give the 
corresponding mono-TPE-substituted BODIPYs (TPEBDP1-2-3). To obtain triple-TPE-substituted BODIPYs, monosubstituted-BODIPYs were reacted with NBS and yielded the corresponding 2,6-dibromo derivatives. In microwave assisted Suzuki couplings, the 2,6-dibromo-substituted BODIPY derivatives are coupled with boronic ester-substituted TPE into 2,6,8tri-TPE-substituted BODIPYs (3TPEBDP1-2-3). For detailed information about procedures see the Supporting Information.

\subsection{Optical characterization}

AIE characterization: To understand the AIE features of the materials, their luminescent behavior upon aggregation in aqueous media were investigated by photoluminescence (PL) spectroscopy. Photoluminescence was collected from a Horiba Fluoromax-4 spectrofluorimeter and excitation wavelength for each sample indicated in section 3.1. For the preparation of samples, dry tetrahydrofuran (THF) was selected as good solvent while water was selected as aggregation-inducing nonsolvent. Stock solution were prepared. Aliquots of the stock solution were transferred to $10 \mathrm{ml}$ vials and appropriate amounts of THF were added with a micropipette. Then, water was added dropwise to each vial with increasing fraction (between $0-90$ vol.\% water). The final mixture concentrations were fixed to $10^{-6} \mathrm{M}$. PL measurements of 10 samples for each compound were performed immediately.

Thin film preparation and optical characterization: Thin films were spin-casted onto fused silica substrates from $10 \mathrm{mg} / \mathrm{mL}$ toluene solutions. The films were deposited via spin-coating to obtain a thickness of $\sim 100 \mathrm{~nm}$, measured with a Dektak profilometer. Photoluminescence was collected from an Andor Shamrock 163 spectrograph coupled with an Andor Newton EMCCD. The PLQY experiments were carried out using an integrating sphere setup. For all PL experiments, samples were excited by using $450 \mathrm{~nm}$ and $520 \mathrm{~nm}$ laser diodes (average power below $1 \mathrm{~mW}$ ). Time-resolved PL measurements were carried out with a time-correlated single photon counting (TCSPC) spectrometer as previously reported [24].

\subsection{OLEDs fabrication and characterization}

OLEDs were fabricated by sandwiching the AIE films between ITO/PEDOT:PSS anodes and Ca/Al cathodes. ITO substrates were cleaned with acetone and isopropanol in an ultrasonic bath and treated in an $\mathrm{O}_{2}$ plasma chamber for 10 min. A $40 \mathrm{~nm}$ layer of PEDOT:PSS (Sigma Aldrich) was spin-coated at $5000 \mathrm{rpm}$ from a $2.8 \mathrm{wt} . \%$ dispersion in water and annealed at $150{ }^{\circ} \mathrm{C}$ for $10 \mathrm{~min}$. The active layer was spin-coated on top of the annealed PEDOT:PSS in $\mathrm{N}_{2}$ environment from the same 10 $\mathrm{mg} / \mathrm{mL}$ toluene solutions used for the optical characterization in the solid state. A Ca/Al (30/200 nm) cathode was thermally evaporated on top. The samples were then encapsulated with a UV curable epoxy resin (OSSILA E132) and measured in air using a Keithley 2400 source meter for both the current measurement and the voltage supply. The optical output of the PLEDs was measured with a calibrated silicon photodiode and the EL spectra were collected with the same spectrometer employed for the PL experiments.

\section{Results and discussion}

\subsection{Aggregation-induced emission characterization}

The photoluminescence spectra of TPEBDPx and 3TPEBDPx series were investigated in THF/water mixtures with increasing water fraction (in vol.\%). By this method, solvent polarity and, corresponding, amount of aggregation were finetuned. 

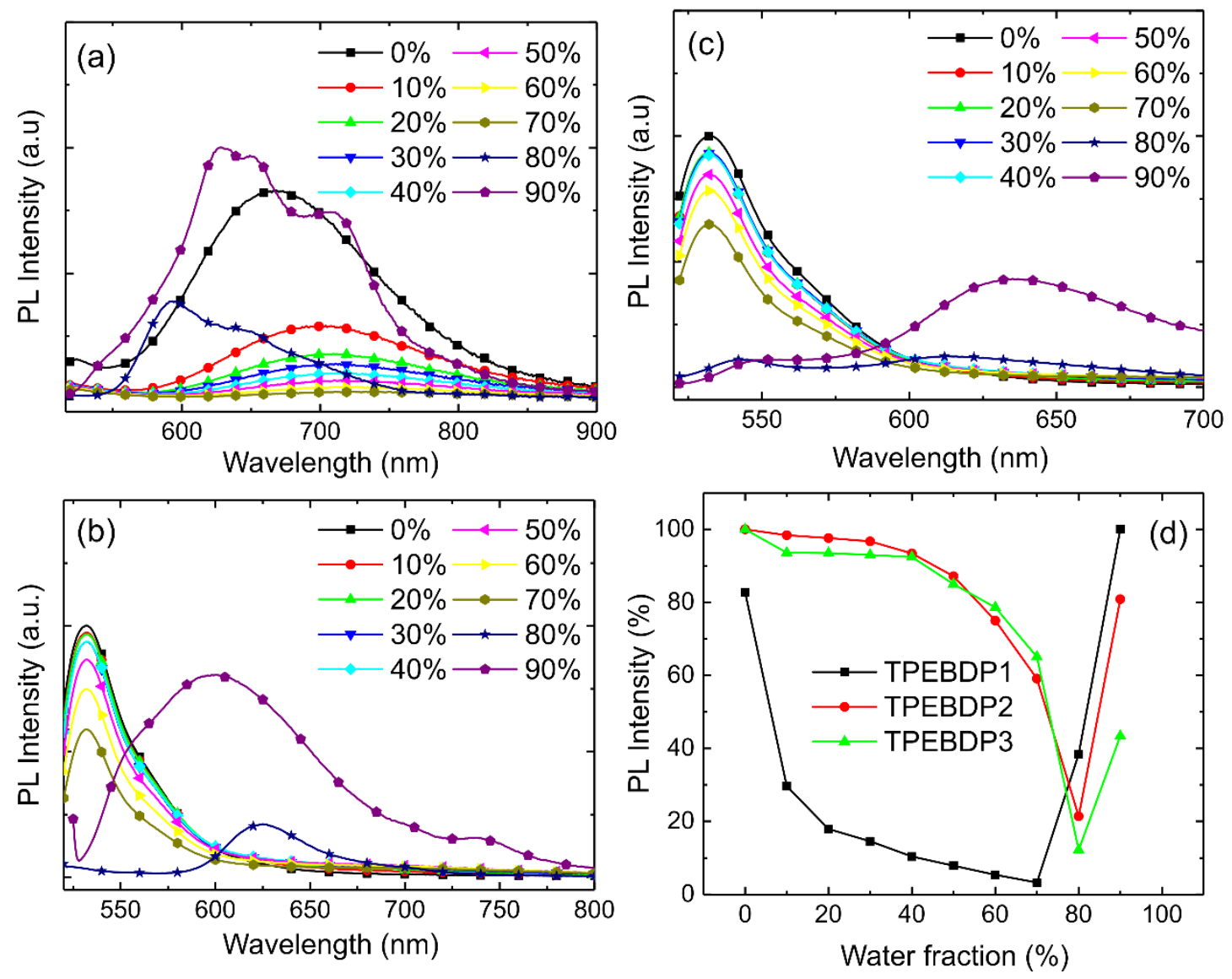

Figure 1. PL spectra of a) TPEBDP1, b) TPEBDP2, c) TPEBDP3 in THF/water mixtures at different water contents; d) The change of PL intensity with increasing water fraction.

The PL spectra of mono-TPE-substituted BODIPYs (TPEBDPx) are shown in Figure 1. TPEBDP2 and TPEBDP3 in THF solutions have an emission around $530 \mathrm{~nm}$ (excitation wavelength: $511 \mathrm{~nm}$ ) due to BODIPY core. With gradual addition of water into THF, the emission is reduced and bathochromically shifted to $609 \mathrm{~nm}$ for TPEBDP2 and $640 \mathrm{~nm}$ for TPEBDP3. Such a large bathochromic shift of PL can also be visually perceived by the color change of solutions, both under daylight and UV illumination (Supporting Information Figure S1). TPEBDP2 has already been reported by Tang et al, who investigated also the effect of solvent polarity and temperature on the emission [16]. Namely, they demonstrated that the spectral changes upon increasing solvent polarity can be explained by the AIE effect and the concomitant formation of twisted intramolecular chargetransfer (TICT) states. As shown in Figure S4 of the Supporting Information, TPEBDP3 emission shows a trend similar to the one reported by Tang when measured in solvents with different polarity. Furthermore, for both TPEBDP2 and TPEBDP3, the molecules start to show the AIE effect at 80 vol.\% water (Figure 1d). However, the starting emission intensity is not fully recovered to its highest value at 90 vol.\% water.

In case of TPEBDP1 (Figure 1a), the emission in THF is peaked at $\sim 670 \mathrm{~nm}$ (excitation wavelength: $501 \mathrm{~nm}$ ). As the water content is increased, the intensity of emission first decreases. Starting from 80 vol.\% water TPEBDP1 shows an obvious AIE effect with a weak blue-shift of the emission $\left(\lambda_{\max e m}: 658 \mathrm{~nm}\right)$. It is likely that the lack of alkyl substituents promotes the formation of aggregates at higher water contents. The maximum of fluorescence is achieved for 90 vol. $\%$ water. 

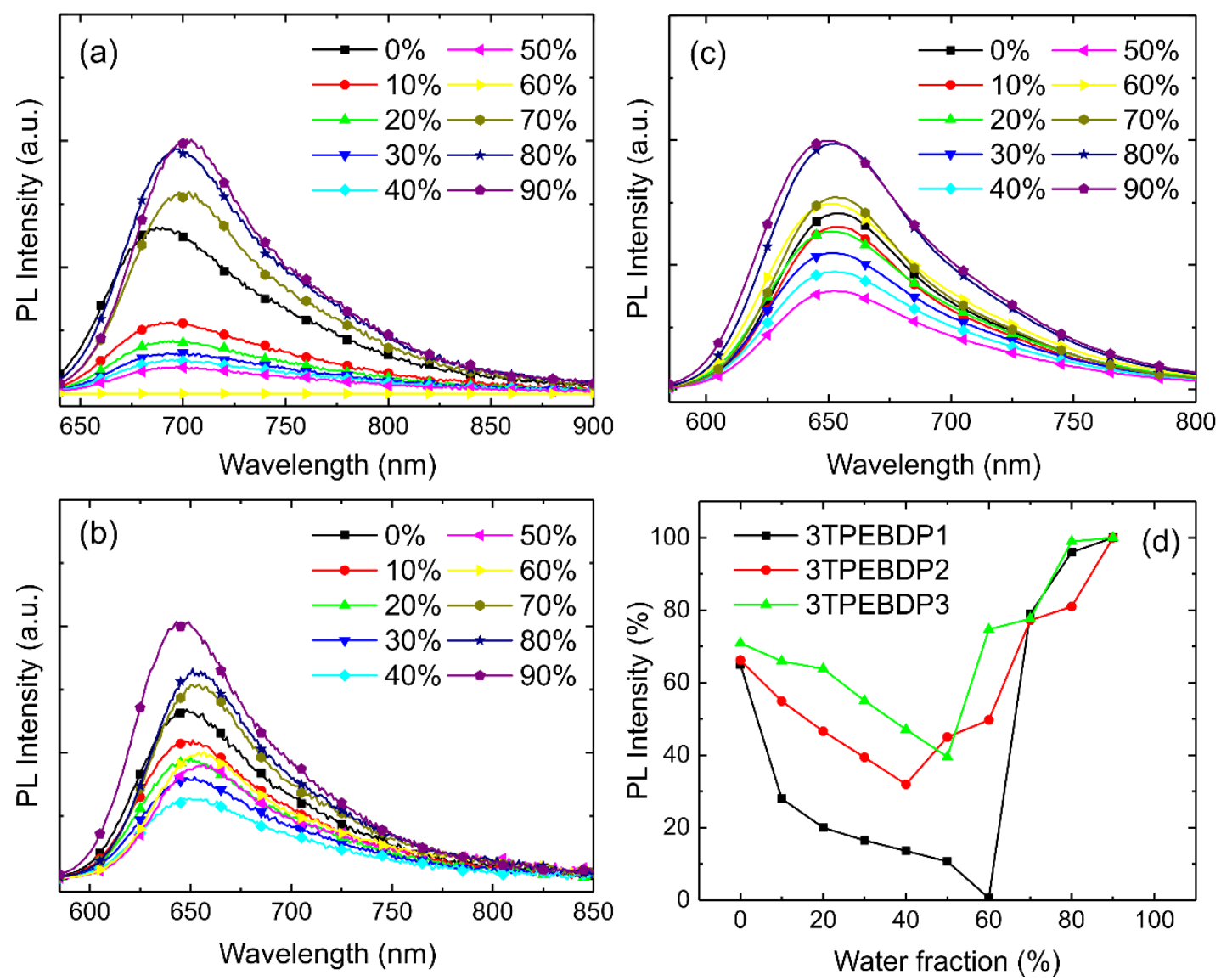

Figure 2. PL spectra of a) 3TPEBDP1, b) 3TPEBDP2, c) 3TPEBDP3 in THF/water mixtures of different water contents; d) The change of PL intensity with increasing water fraction.

The PL spectra of the three tri-TPE-substituted BODIPYs (3TPEBDPx) are shown in Figure 2. The emission maxima in THF are observed at 689,646 , and $648 \mathrm{~nm}$ respectively. With gradual increase of water content in the THF/water mixture, PL intensities decreased up to $60 \mathrm{vol} . \%, 40 \mathrm{vol} . \%$, and 50 vol.\% water fractions respectively for 3TPEBDP $1-2-3$. Continued water addition causes aggregate formation which results in increased PL intensities with reaching the highest value at 90 vol. $\%$ water. The PL intensity of the aggregated samples (90\% water) is ca. 1.53 times higher for 3TPEBDP1, if compared to pure THF solution, 1.50 times higher for 3TPEBDP2, and 1.40 times higher for 3TPEBDP3, thus showing a clear, but moderate AIE effect. (see Figure 2d)

The addition of two extra TPE units to the TPEBDPx series induces a more pronounced AIE effect, as expected due to the increased number of rotor units if compared to the mono-TPE-substituted analogues.

\subsection{Optical properties in the solid state}

The solid-state absorption and photoluminescence properties of the TPEBDPx and 3TPEBDPx series was carried out with thin films deposited from $10 \mathrm{mg} / \mathrm{mL}$ toluene solutions by spin coating. Toluene as solvent allows to obtain uniform films. Homogeneity of the emitting layer is indeed an important prerequisite to fabricate efficient light-emitting devices. The absorption and PL spectra of these films are presented in Figure 3. 

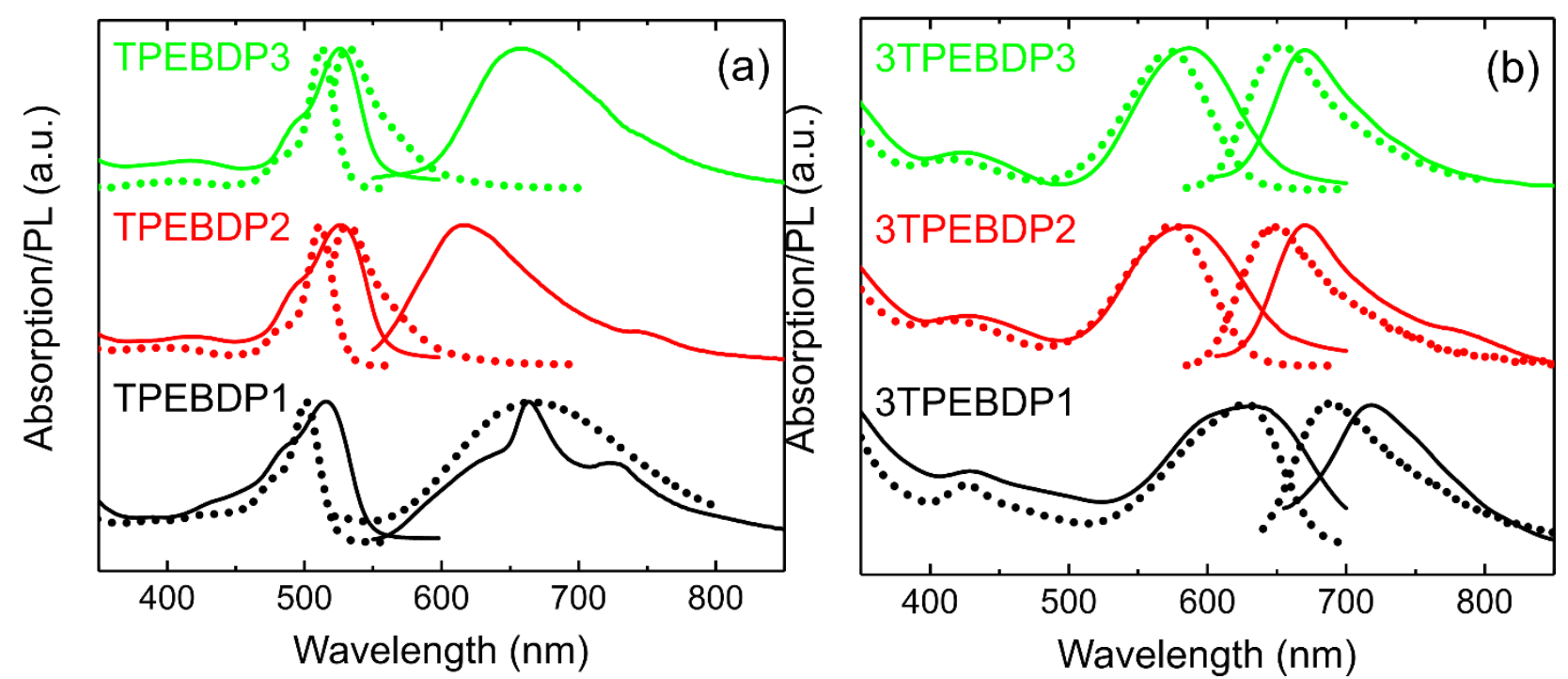

Figure 3. Absorption and emission of TPEBDPx and 3TPEBDPx in diluted THF solution (dotted lines) and solid-state thin films (full lines). For the PL experiments, samples were excited with a $520 \mathrm{~nm}$ laser diode (average power $<1 \mathrm{~mW}$ ).

The absorption spectra of the mono-TPE substituted molecules (TPEBDPx) are dominated by the absorption component of the BODIPY moiety peaking at $\sim 520 \mathrm{~nm}$. Most likely due to aggregation, we also observe a broadening and a $10 \mathrm{~nm}$ red-shift of these bands when moving from solution to film. Whereas in solution TPEBDP2 and TPEBDP3 exhibit a PL emission peaked at $525 \mathrm{~nm}$ with full width at half maximum (FWHM) of only $30 \mathrm{~nm}$, which is typical of 2,6-unsubstituted BODIPYs [25], the PL spectra from the corresponding spin-casted films exhibit broad PL spectra with maxima at $615 \mathrm{~nm}$ for TPEBDP2 and 650 $\mathrm{nm}$ for TPEBDP3. Such a substantial $(>100 \mathrm{~nm})$ Stokes shift is most likely the result of aggregation in the solid-state, in analogy to what is observed in water:THF solutions with large water (non-solvent) percentages (Figure 1). Regarding the $\alpha$ unsubstituted TPEBDP1, we note that both solution and film present a PL peaking at $670 \mathrm{~nm}$.

Looking at the tri-TPE substituted molecules (3TPEBDPx), we observe a substantial red-shift of the absorption and emission spectra compared to the TPEBDPx series, reaching up to $120 \mathrm{~nm}$ for 3TPEBDP1. Furthermore, in contrast to what is observed with TPEBDP1-2-3, both absorption and emission spectra of the 3TPEBDP1-2-3 molecules in the solid state (Figure 3 ) are only slightly red-shifted $(10-20 \mathrm{~nm})$ and broader compared to those measured in solution. Namely, the absorption spectra are dominated by a broad band in the $500-650 \mathrm{~nm}$ spectral region and maxima at $580 \mathrm{~nm}$ for 3TPEBDP2 and 3, and in the $550-700 \mathrm{~nm}$ range with maximum at $635 \mathrm{~nm}$ for 3TPEBDP1. Concerning the PL, spectra measured from 3TPEBDP2 and 3 films peak at $670 \mathrm{~nm}$, whereas the PL of 3TPEBDP3 shows a maximum in the NIR at $720 \mathrm{~nm}$.

To verify the presence of AIE effect, we measured also the PL quantum efficiency $\Phi_{\mathrm{F}}$ of each film and collected the corresponding values in Table 1 .

Table 1. PL quantum efficiency $\left(\Phi_{F}\right)$, highest occupied molecular orbital $(H O M O)$ and lowest occupied molecular orbital level $(L U M O)$ energy levels. PL efficiency values are to be considered with a $10 \%$ uncertainty on the absolute value. For the PL efficiency experiments, samples were excited with a $520 \mathrm{~nm}$ laser diode (average power below $1 \mathrm{~mW}$ ). HOMO values were determined by atmospheric pressure photo-electron spectroscopy $(\mathrm{AC}-2), \mathrm{LUMO}=\mathrm{Eg}(\mathrm{opt})$ HOMO(AC-2), See Eg(opt) values in Table S2.

\begin{tabular}{ccccc}
\hline Compound & $\Phi_{F}$ sol (\%) & $\Phi_{F}$ film (\%) & HOMO $(\mathrm{eV})$ & LUMO $(\mathrm{eV})^{*}$ \\
\hline TPEBDP1 & 8 & 10 & -5.63 & -3.39 \\
TPEBDP2 & 4 & 4 & -5.84 & -3.64 \\
TPEBDP3 & 4 & 5 & -5.82 & -3.59 \\
3TPEBDP1 & 47 & 10 & -5.69 & -3.96 \\
3TPEBDP2 & 29 & 39 & -5.69 & -3.82 \\
3TPEBDP3 & 44 & 48 & -5.65 & -3.78 \\
\hline
\end{tabular}

With the exception of 3TPEBDP1, all oligomers show similar or slightly higher $\Phi_{\mathrm{F}}$ for the solid-state films if compared 
to the solution values. Notably, 3TPEBDP2 and 3 in the solid-state show $\Phi_{\mathrm{F}}$ of $39 \%$ and $48 \%$, respectively, which are relatively high and not easily attainable with conventional molecular chromophores when spin-coated from solution as neat films. For completeness, we report in Figure S10 and Table S2 also the PL spectra and corresponding $\Phi_{\mathrm{F}}$ obtained from films deposited from THF solutions. By comparing the spectra from the films obtained from toluene (Figure 3) and those obtained from THF (Figure S10), no major variations can be detected. Furthermore, for TPEBDPx molecules, the $\Phi_{\mathrm{F}}$ values reported in Table S2 for the films spin-coated from THF are similar to those obtained from toluene (Table 1). On the contrary, due to additional TPE units for the 3TPEBDPx dyes, we note a remarkable variation, especially for the most efficient 3TPEBDP2 and 3 dyes. Namely, for the films obtained from THF solutions we obtained $\Phi_{\mathrm{F}}$ values reaching up to $68 \%$ for 3 TPEBDP2 and $98 \%$ for 3 TPEBDP3. However, such films were highly inhomogeneous and, by exciting different areas of the sample and different samples, we measured efficiencies below $40 \%$ for both molecules.

\subsection{Aggregation-induced emission in F8BT blends}

Given that we obtained the highest PL efficiencies from the 3TPEBDPx series, with 3TPEBDP2 and 3 benefitting from the AIE effect in the solid-state, we decided to focus on these materials to fabricate OLEDs. Furthermore, 3TPEBDPx molecules, here presented for the first time, are attractive also because a good fraction of their emission $(78 \%, 43 \%$ and $35 \%$ for 3TPEBDP1,2 and 3, respectively) is in the NIR (> $700 \mathrm{~nm}$ ). In this spectral range, PL efficiencies up to $50 \%$ in the solid state (Table 1) are, in general, difficult to achieve, mainly due to ACQ and the so-called "Energy-gap law" [26].

To maximize the performance of the final devices, we incorporated the 3TPEBDPx molecules in a solid-state blend with F8BT, as well-established green emitting semiconductor polymer [2]. Among the plethora of commercially available semiconducting polymers, we selected F8BT as polymer host for mainly two reasons: first of all, its emission perfectly overlaps with the absorption of 3TPEBDPx, thereby affording efficient energy transfer to the red/NIR emitting moieties. Secondly, and most importantly, the HOMO $(-6.0 \mathrm{eV})$ and LUMO $(-3.3 \mathrm{eV})$ of F8BT enable the formation of a 'so-called' type I or straddling heterojunction when blended with 3TPEBDPx molecules, whose energies of the frontier molecular orbitals are collected in Table 1. Namely, the 3TPEBDPx narrower energy bandgap is completely encompassed by the F8BT larger one, so as to form a straddling heterojunction, which favors exciton localization and prevents exciton dissociation.

Prior to integration of the blends into OLEDs, we characterized the optical properties of such solid-state mixtures, also to identify if the AIE effect is conserved. To this aim, we investigated different concentrations of 3TPEBDPx molecules in F8BT, to find out that the best results in terms of quenching of the residual F8BT emission and PL efficiency are obtained, for all 3TPEBDPx moieties, from 3TPEBDPx:F8BT blends with $1 \mathrm{w} / \mathrm{w} \%$ of 3TPEBDPx loading. The absorption and corresponding emission spectra of such films are illustrated in Figure 4, whereas $\Phi_{\mathrm{F}}$ values are collected in Table 2.

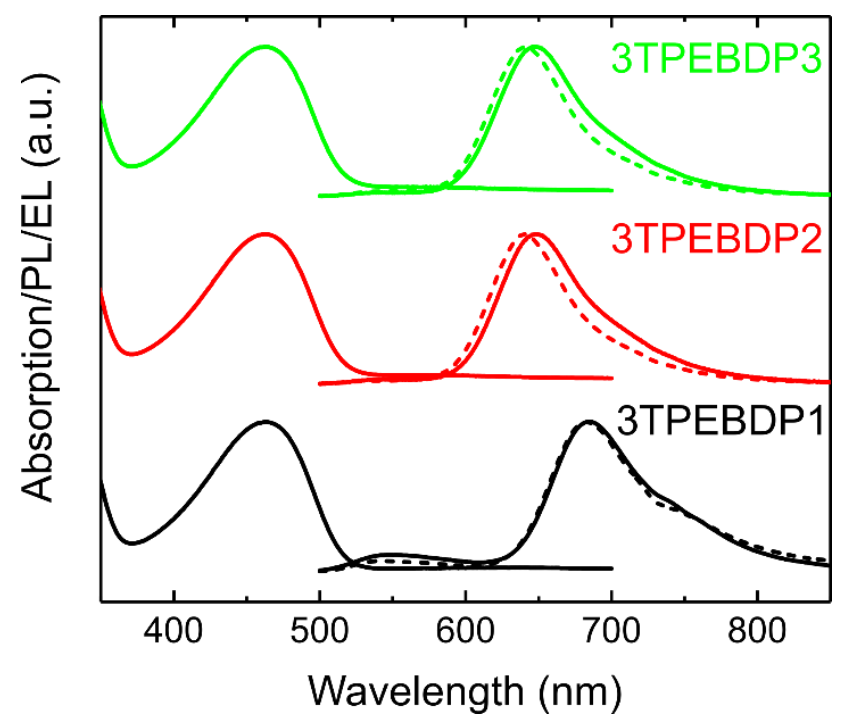

Figure 4. Absorption, PL and EL spectra (dashed lines) of 3TPEBDPx:F8BT blends with $1 \mathrm{w} / \mathrm{w} \%$ of 3 TPEBDPx loading. The absorption spectra of the blends are dominated by the F8BT band peaking at $460 \mathrm{~nm}$. 
Table 2. PL quantum efficiency $\left(\Phi_{F}\right)$ of neat films and 0.1 and $1 \mathrm{w} / \mathrm{w} \% 3$ TPEBDP1:F8BT blends, PL lifetime and calculated radiative $\left(k_{R}\right)$ and non-radiative $\left(k_{N R}\right)$ rate. PL efficiency values are to be considered with a $10 \%$ uncertainty on the absolute value. For the PL efficiency experiments on the blends, samples were excited with a $450 \mathrm{~nm}$ laser diode (average power below $1 \mathrm{~mW}$ ) to selectively excite the F8BT matrix

\begin{tabular}{ccccc}
\hline film & $\Phi_{F}$ film $(\%)$ & $\tau(n s)$ & $k_{R}\left(n s^{-1}\right)$ & $k_{N R}\left(n s^{-1}\right)$ \\
\hline neat 3TPEBDP1 & 10 & 0.68 & 0.15 & 1.33 \\
1\% 3TPEBDP1 & 47 & 2.7 & 0.17 & 0.20 \\
\hline neat 3TPEBDP2 & 39 & 1.01 & 0.39 & 0.60 \\
1\% 3TPEBDP2 & 96 & 4.29 & 0.22 & 0.01 \\
\hline neat 3TPEBDP3 & 48 & 1.34 & 0.36 & 0.38 \\
1\% 3TPEBDP3 & 100 & 4.56 & 0.22 & 0 \\
\hline
\end{tabular}

By looking at the absorption spectra in Figure 4, we note that the extinction profile is dominated by the F8BT absorption. Nonetheless, at only $1 \mathrm{w} / \mathrm{w} \%$ of 3TPEBDPx concentration, the emission from the F8BT host is almost completely quenched for all three combinations, thanks to the efficient resonant energy transfer from the polymeric host to the emitters. Among the three molecules, 3TPEBDP1 is the one in which the ratio between the F8BT and acceptor PL is higher.

However, not only is 3TPEBDP1:F8BT the blend exhibiting the most prominent green emission from the host, but also, looking at Table 2, 3TPEBDP1:F8BT films are those exhibiting the lowest $\Phi_{\mathrm{F}}$. This means that, although $\Phi_{\mathrm{F}}$ values increase from $10 \%$ to $47 \%$ when embedded in F8BT (Table 2), aggregation quenching affects the 3TPEBDP1 PL performance also when in blend with F8BT and prevents the attainment of higher PL efficiencies. On the other hand, 3TPEBDP2 and 3TPEBDP3 $1 \mathrm{w} / \mathrm{w} \%$ blends display $\Phi_{\mathrm{F}} \sim 100 \%$, and almost no residual green emission from F8BT. We tested also higher doping levels for 3TPEBDP2 and 3TPEBDP3 (up to $50 \mathrm{w} / \mathrm{w} \%$ ), but we observed a decrease of the efficiency when the 3TPEBDPx content exceeded $1 \%$. Therefore, we conclude that the $1 \% 3$ TPEBDP2 and 3TPEBDP3 concentration is optimal for maximizing both the PLQY and energy transfer from F8BT.

We have also measured the PL decay lifetimes of both the bare 3TPEBDPx films and blends. The evolution of the PL decay at varying 3TPEBDPx concentration is illustrated in Figure S6, whereas the decay lifetimes $(\tau)$ and the radiative ( $\left.k_{R}\right)$ and non-radiative $\left(\mathrm{k}_{\mathrm{NR}}\right)$ decay rates are reported in Table 2 . We obtained $\mathrm{k}_{\mathrm{R}}$ and $\mathrm{k}_{\mathrm{NR}}$ via the relations $\Phi_{\mathrm{F}}=\mathrm{k}_{\mathrm{R}} /\left(\mathrm{k}_{\mathrm{R}}+\mathrm{k}_{\mathrm{NR}}\right)$ and $\tau=$ $1 /\left(k_{R}+k_{N R}\right)$. Importantly, we note that the $k_{R}$ and $k_{N R}$ values reported in Table 2 for the blends are probably less accurate compared to those of the neat films, as part of the emitted photons that account for the corresponding $\Phi_{\mathrm{F}}$ originate from the unquenched F8BT matrix (Figure 4). However, such an approximation in the calculation of $\mathrm{k}_{\mathrm{R}}$ and $\mathrm{k}_{\mathrm{NR}}$ is reasonably plausible, given the substantial increase of $\tau$ and $\Phi_{\mathrm{F}}$ when 3TPEBDPx molecules are embedded in F8BT.

Looking first at the neat 3TPEBDPx films, Table 2 shows that $\mathrm{k}_{\mathrm{R}}$ for 3TPEBDP1 is about half compared to the one of 3TPEBDP2 and 3TPEBDP3. Such a lower radiative rate further supports the discussion above, confirming that intermolecular interactions, and therefore ACQ, are more effective in 3TPEBDP1 films compared to the others. Furthermore, 3TPEBDP1 films also show non-radiative rates that are more than twice as high compared to the other compounds. This suggests a limited AIE-induced enhancement of 3TPEBDP1 PL efficiency, at least in the solid state.

Moving to the blends, as suggested already from the increasing $\Phi_{\mathrm{F}}$, the AIE-effect is verified for all systems, i.e. $\mathrm{k}_{\mathrm{NR}}$ is reduced to a much larger extent compared to $\mathrm{k}_{\mathrm{R}}$. For 3TPEBDP2 and 3TPEBDP3, this conclusion is somehow trivial, given that the $\Phi_{\mathrm{F}}$ in $\mathrm{F} 8 \mathrm{BT}$ is $\sim 100 \%$, undoubtedly meaning that all non-radiative exciton recombination processes, including intermolecular rotations, are precluded more efficiently than in the neat films. However, it is worth highlighting that, although $\mathrm{k}_{\mathrm{NR}} \sim 0$, also $\mathrm{k}_{\mathrm{R}}$ decreases by a factor of about two, suggesting that intermolecular interactions, either in the ground state or in the excited state (excimer or exciplex formation), are probably enhanced in the blends compared to the neat film.

Concerning F8BT:3TPEBDP1 blends, $\mathrm{k}_{\mathrm{NR}}$ is reduced even more dramatically from $1.3 \mathrm{~ns}^{-1}$ down to $0.2 \mathrm{~ns}^{-1}$, meaning that the effectiveness of the non-radiative processes responsible for the quenching of the $\Phi_{\mathrm{F}}$ of $3 \mathrm{TPEBDP} 1$ from $47 \%$ in solution to $10 \%$ in film (Table 1) is mitigated when 3TPEBDP1 is diluted in a F8BT matrix. On the other hand, $\mathrm{k}_{\mathrm{R}}$ exhibits only a slight increase from 0.15 to $0.17 \mathrm{~ns}^{-1}$ in the $1 \%$ blend, although the residual F8BT emission might also account for such a little variation compared to the factor of two decrease of $\mathrm{k}_{\mathrm{R}}$ observed for 3TPEBDP2 and 3TPEBDP3. This results also mean that, in analogy to what is observed for 3TPEBDP2 and 3TPEBDP3, dilution in F8BT does not suppress intermolecular interactions between 3TPEBDP1 molecules. Besides, electronic interactions between 3TPEBDPx and F8BT chains cannot be ruled out either.

\subsection{OLEDs}

Finally, given the extremely high PL efficiencies we obtained from the F8BT:3TPEBDPx blends, we used them to 
fabricate OLEDs, with the architecture and deposition techniques described in the experimental section. The electroluminescence (EL) spectra of such blends are reported in Figure 4, whereas the density-voltage-radiance (JVR) and External Quantum Efficiency (EQE) versus current density curves are reported in Figure 5. OLEDs characteristics are summarised in Table 3.
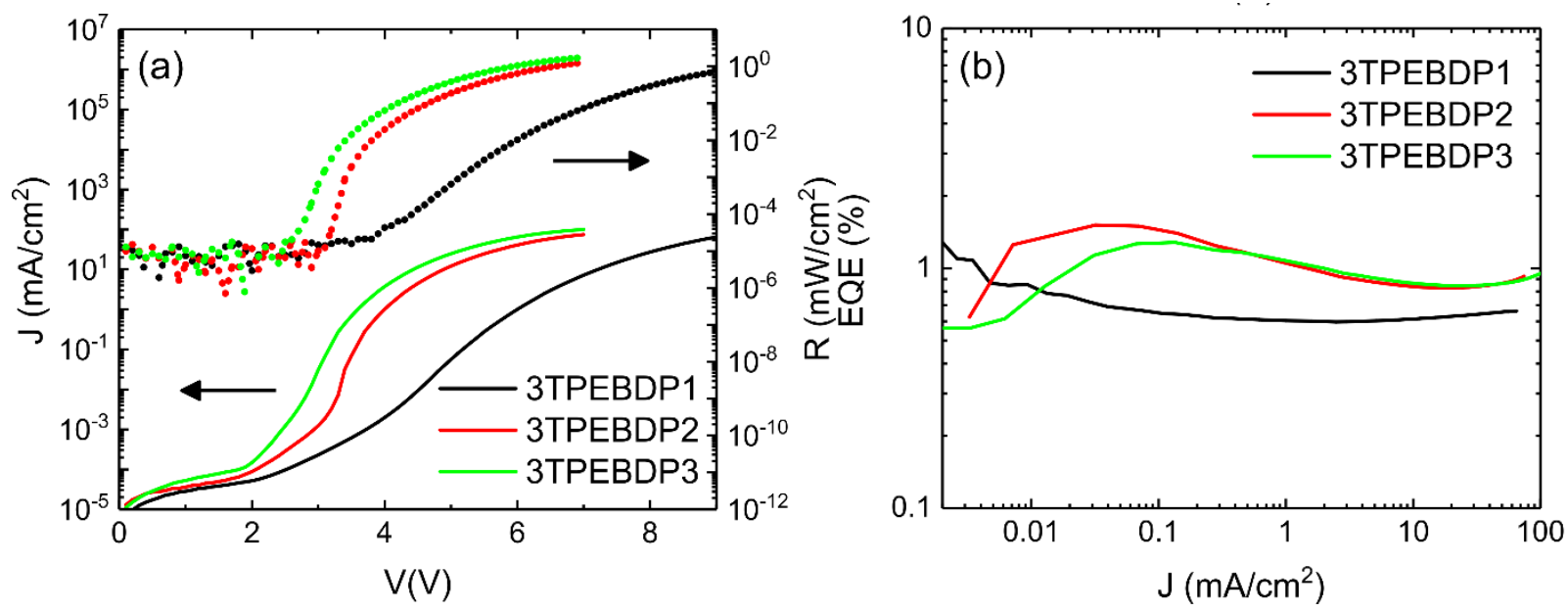

Figure 5. JVR curves (a) and EQE versus current density plot (b) of OLEDs incorporating F8BT: 3TPEBDPx 1\% blends.

Table 3. OLEDs summary of the device characteristics of F8BT:3TPEBDPx OLEDs.

\begin{tabular}{ccccc}
\hline Sample & $\begin{array}{c}V_{O N} \\
(\mathrm{~V})\end{array}$ & $\begin{array}{c}\text { Radiance } \\
\left(\mathrm{mW} / \mathrm{cm}^{2}\right)\end{array}$ & $\begin{array}{c}<E Q E_{M A X}>^{\mathrm{a})} \\
(\%)\end{array}$ & $\begin{array}{c}\left.E Q E_{M A X} \mathrm{~b}\right) \\
(\%)\end{array}$ \\
\hline $1 \%$ 3TPEBDP1 & $4.3 \pm 0.6$ & $0.91 \pm 0.55$ & $0.51 \pm 0.37$ & 1.29 \\
$1 \%$ 3TPEBDP2 & $3.2 \pm 0.3$ & $1.60 \pm 0.18$ & $1.47 \pm 0.21$ & 1.82 \\
$1 \%$ 3TPEBDP3 & $3 \pm 0.2$ & $1.62 \pm 0.41$ & $1.16 \pm 0.13$ & 1.31
\end{tabular}

a)Average maximum EQE. b) Maximum EQE

By looking at Figure 4, we observe that EL spectra of the blends show only a slight $(\sim 5 \mathrm{~nm})$ blue-shift compared to the PL ones. Furthermore, as for the PL, the EL from the F8BT host is efficiently quenched in favor of the red/NIR emission from the 3TPEBDPx dopant. In terms of device performance, we obtained the best efficiencies from the F8BT:3TPEBDP2 blend, which exhibited an average EQE of $1.47 \%$, with a peak value at $1.82 \%$. Such values are remarkably high, considering that we achieved them from solution-processed devices, not optimized for charge injection/transport and photon out-coupling. Moreover, the maximum radiance reaches up to $2 \mathrm{~mW} / \mathrm{cm}^{2}$ with F8BT:3TPEBDP2 devices, with a turn-on voltage as low as 3 $\mathrm{V}$.

Although slightly less efficient (maximum EQEs up to $1.3 \%$ ), also devices incorporating the F8BT:3TPEBDP3 blend exhibited radiances up to $2 \mathrm{~mW} / \mathrm{cm}^{2}$, and $\mathrm{V}_{\mathrm{ON}} \sim 3 \mathrm{~V}$. Notably, whereas the EL $\mathrm{V}_{\mathrm{ON}}$ of both F8BT:3TPEBDP2 and F8BT:3TPEBDP3 blends match the one of the neat F8BT devices (see Supporting Information, Figure S11), the EQE and radiance values reported in Table 3 exceed those we measured from the undoped F8BT OLEDs, for which the maximum EQE and radiance we measured are $0.86 \%$ and $1.1 \mathrm{~mW} / \mathrm{cm}^{2}$, respectively.

\section{Conclusions}

In summary, we have synthesized red/NIR emitting mono-TPE-substituted and tri-TPE-substituted BODIPY derivatives and investigated their AIE properties. Due to the high solid-state emission of tri-TPE-substituted materials, OLED devices performed and attained EQE values exceeding $1 \%$ from NIR emitting 3TPEBDP1-2-3/F8BT blends as "metal-free" active 
layer. To the best of our knowledge, such efficiencies, together with low turn-on voltage (2.5-4V) and radiances reaching up to $2 \mathrm{~mW} / \mathrm{cm}^{2}$, are unprecedented for AIE OLEDs emitting in this spectral range $(600-800 \mathrm{~nm})$ and fabricated via solution processing. Further optimization can lead to progresses of these emitters for the use of biomedical and communication purposes.

Acknowledgments This project has received funding from the European Community's Seventh Framework Programme (FP7/2007-2013) under the Grant Agreement ${ }^{\circ} 607585$.

S.B. and S.A. thanks Stefan Türk from MPI, Anke Helfer and Sylwia Adamczyk from Bergische Universität Wuppertal for mass and AFM characterizations.

S.B. and S.A. thank Dr. Michael Forster from BUW for the helpful discussions.

S.B. thanks Prof. Ullrich Scherf and Bergische Universität Wuppertal for the financial support.

F.C. is a Royal Society Wolfson Research Merit Award holder

Conflict of interest The authors declare that they have no conflict of interest.

Supporting information The supporting information consist of detailed synthesis, NMR data, AIE measurements images, solvatochromism measurements of TPEBDP1, low temperature PL of 3TPEBDPx, PL decays of 3TPEBDPx and F8BT:3TPEBDPx films by TCSPC, AFM images of 3TPEBDPx, Optical properties of TPEBDPx and 3TPEBDPx in THF solution and undoped F8BT OLED device characterization. The supporting information is available online at $\mathrm{http}$ ://chem.scichina.com and http://link.springer.com/journal/11426. The supporting materials are published as submitted, without typesetting or editing. The responsibility for scientific accuracy and content remains entirely with the authors.

1. Boens N, Leen, W, Dehaen W. Fluorescent indicators based on BODIPY. Chem. Soc. Rev, 2012, 41: 1130-1172, DOI: 10.1039/C1CS15132K

2. Zampetti A, Minotto A, Squeo BM, Gregoriou VG, Allard S, Scherf U, Chochos CL, Cacialli F. Highly efficient solid-state Near-infrared Organic Light-Emitting Diodes incorporating A-D-A Dyes based on $\alpha, \beta$-unsubstituted ''BODIPY'” Moieties. Sci. Rep, 2017, 7:1611, DOI: 10.1038/s41598017-01785-2

3. Murto P, Minotto A, Zampetti A, Xu X, Andersson MR, Cacialli F, Wang E. Triazolobenzothiadiazole-Based Copolymers for Polymer Light Emitting Diodes: Pure Near-Infrared Emission via Optimized Energy and Charge Transfer. Adv. Opt. Mater, 2016, 4: 2068-2076, DOI: 10.1002/adom.201600483

4. Marechal E. Polymeric dyes- synthesis, properties and uses. Prog. Org. Coat, 1982, 10: 251-287, DOI: 10.1016/0300-9440(82)80022-2

5. Ullrich G, Ziessel R, Harriman A. The chemistry of fluorescent BODIPY dyes: versatility unsurpassed. Angew. Chem. Int. Ed, 2008, 47: 1184-1201, DOI: 10.1002/anie.200702070

6. Louder A, Burgess K. BODIPY dyes and their derivatives: syntheses and spectroscopic properties. Chem. Rev, 2007,107: 4891-4932, DOI: $10.1021 / \mathrm{cr} 078381 \mathrm{n}$

7. Ziessel R, Ulrich G,Harriman A. The chemistry of BODIPY: A new El Dorado for fluorescence tools. New J. Chem, 2007, 31(4): 496-501, DOI: 10.1039/B617972J

8. Chen M, Li L, Nie H, Tong J, Yan L, Xu B, Sun JZ, Tian W, Zhao Z, Qin A, Tang BZ. Tetraphenylpyrazine-based AIEgens: facile preparation and tunable light emission. Chem. Sci, 2015, 6: 1932-1937, DOI: 10.1039/C4SC03365E

9. Dong W, Pina J, Pan Y, Preis E, Melo JS, Scherf U. Polycarbazoles and polytriphenylamines showing aggregation-induced emission (AIE) and intramolecular charge transfer (ICT) behavior for the optical detection of nitroaromatic compounds. Polymer, 2015, 76: 173-181, DOI:10.1016/j.polymer.2015.08.064

10. Zhao Z, Chen S, Lam JWY, Wang Z, Lu P, Mahtab F, Sung HHY, Williams ID, Ma Y, Kwok HS, Tang BZ. Pyrene-substituted ethenes: aggregationenhanced excimer emission and highly efficient electroluminescence. J. Mater. Chem, 2011, 21: 7210-7216, DOI: 10.1039/C0JM04449K

11. Zhao Z, Chen S, Lam JWY, Lu P, Zhong Y, Wong KS, Kwok HS, Tang BZ. Creation of highly efficient solid emitter by decoration pyrene core with AIE-active tetraphenylethene peripheries. Chem. Commun, 2010, 46: 2221-2223, DOI: 10.1039/B921451H

12. Zhao Q, Zhang S, Liu Y, Mei J, Chen S, Ping L, Qin A, Ma Y, Sun JZ, Tang BZ. Tetraphenylethenyl-modified perylene bisimide: aggregation-induced red emission, electrochemical properties and ordered microstructures. J. Mater. Chem, 2012, 22: 7387-7394, DOI: 10.1039/C2JM16613E

13. Yuan WZ, Lu P, Chen S, Lam JWY, Wang Z, Liu Y, Kwok HS, Ma Y, Tang BZ. Changing the behavior of chromophores from aggregation-caused quenching to aggregation-induced emission: development of highly efficient light emitters in the solid state. Adv. Mater, 2010, 22: 2159-2163, DOI: 10.1002/adma.200904056

14. Luo J, Xie J, Lam JWY, Cheng L, Chen H, Qiu C, Kwok HS, Zhan X, Liu Y, Zhu D, Tang BZ. Aggregation-induced emission of 1-methyl-1,2,3,4,5pentaphenylsilole. Chem. Commun, 2001, 18: 1740, DOI: 10.1039/B105159H

15. Mei J, Hong Y, Lam JWY, Qin A, Tang Y, Tang BZ. Aggregation-Induced Emission: The whole is more brilliant then the parts. Adv. Mater, 2014, 26: 5429-5479, DOI: 10.1002/adma.201401356

16. Gomez-Duran CFA, Hu R, Feng G, Li T, Bu F, Arseneault M, Liu B, Pena-Cabrera E, Tang BZ. Effect of AIE substituents on the Fluorescence of tetraphenylethene-containing BODIPY Derivatives. ACS Appli. Mater. Interfaces, 2015, 7(28): 15168-15176, DOI: 10.1021/acsami.5b05033

17. Hu R, Lager E, Aguilar-Aguilar A, Liu J, Lam JWY, Sung HHY, Williams ID, Zhong Y, Wong KS, Pena-Cabrera E, Tang BZ. Twisted intramolecular charge transfer and aggregation-induced emission of BODIPY derivatives. J. Phys. Chem. C, 2009, 113: 15845-45853, DOI: 10.1021/jp902962h

18. Baglan M, Ozturk S, Gur B, Meral K, Bozkaya U, Bozdemir OA, Atilgan S. Novel phenomena for aggregation induced emission enhancement: highly fluorescent hydrophobic TPE-BODIPY couples in both organic and aqeous media. RSC. Advances, 2013, 3: 15866-15874, DOI:10.1039/c3ra40791h

19. Li Q, Qian Y. Aggregation induced emission enhancement and cell imaging of novel (carbazol-N-yl) triphenylamine-BODIPY. New J. Chem, 2016, 40: 7095-7101, DOI: 10.1039/c6nj01495j

20. Zhensheng L, Chen Y, Lv X, Fu WF. A tetraphenylethene-decorated BODIPY monomer/dimer with intense fluorescence in various matrices. New J. Chem, 2013, 37: 3755-3761, DOI: 10.1039/c3nj00703k 
21. Hu R, Gomez-Duran GFA, Lam JWY, Belmonte-Vazquez JL, Deng C, Chen S, Ye R, Pena-Cabrera E, Zhong Y, Wong KS, Tang BZ. Synthesis, Solvatochromism, Aggregation-Induced Emission and Cell Imaging of Tetraphenylethene-Containing BODIPY Derivatives with Large Stokes Shift. Chem. Commun, 2012, 48: 10099-10101, DOI: 10.1039/C2CC35188A

22. Zhao Z, Chen B, Geng J, Chang Z, Aparicio-Ixta L, Nie H, Goh CC, Ng LG, Qin A, Ramos-Ortiz G, Liu B, Tang BZ. Red Emissive Biocompatible Nanoparticles from Tetraphenylethene-Decorated BODIPY Luminogens for Two-Photon Excited Fluorescence Cellular Imaging and Mouse Brain Blood Vascular Visualization. Part. Part. Syst. Charact, 2014, 31: 481-491, DOI: 10.1002/ppsc.201300223

23. Gao H, Gao Y, Wang C, Dehua H, Xie Z, Liu L, Yang B, Ma Y. Anomalous Effect of Intramolecular Charge Transfer on the Light Emitting Properties of BODIPY. ACS. Appl. Mater. Interfaces, 2018, DOI:10.1021/acsami.7b13444

24. Petrozza A, Brovelli S, Michels JJ, Anderson HL, Friend RH, Silva C, Cacialli F. Control of Rapid Formation of Interchain Excited States in SugarThreaded Supramolecular Wires. Adv. Mater, 2008, 20: 3218-3223, DOI:10.1002/adma.200800007

25. Tram K, Yan H, Jenkins HA, Vassiliev S, Bruce D. The synthesis and crystal structure of unsubstituted 4,4-difluoro-4-bora-3a,4a-diaza-s-indacene (BODIPY). Dyes Pigm, 2009, 82: 392-395, DOI: 10.1016/j.dyepig.2009.03.001

26. Englman R, Jortner J. The energy gap law for radiationless transitions in large molecules. Mol. Phys, 1970,18: 145-164, DOI: 10.1080/00268977000100171

\section{Table of Contents graphic}

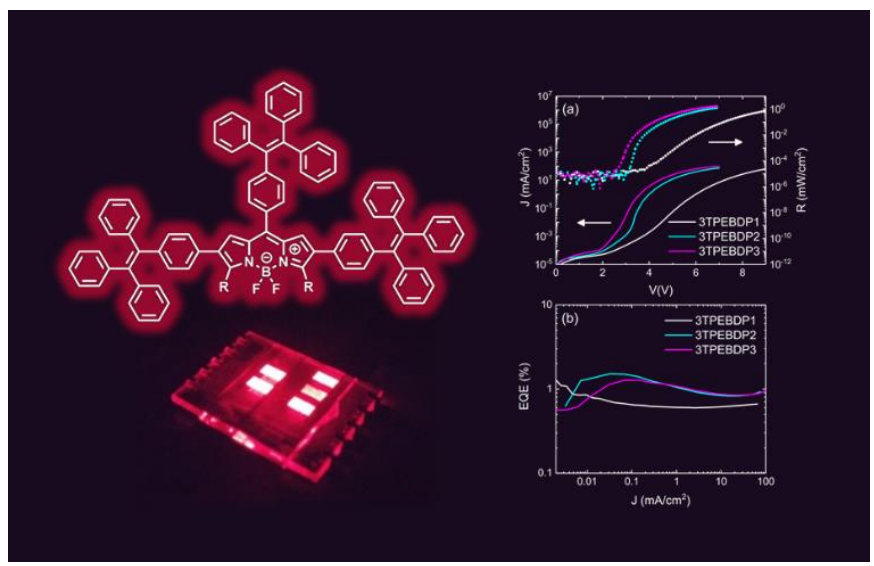

